\title{
Magnon-mediated magnetoresistance in layered manganites
}

\author{
Antonio Hernando, ${ }^{1}$ Raquel Cortés-Gil, ${ }^{2}$ Daniel González-Merchante, ${ }^{2}$ María Hernando, ${ }^{2}$ José M. Alonso, ${ }^{1,3}$ \\ Miguel A. García, ${ }^{1,4}$ José L. Martínez, ${ }^{3}$ Luisa Ruiz-González, ${ }^{2, *}$ and José M. González-Calbet ${ }^{2,5, \dagger}$ \\ ${ }^{1}$ Instituto de Magnetismo Aplicado, UCM-CSIC-ADIF, P.O. Box 155, 28230 Las Rozas de Madrid, Spain \\ ${ }^{2}$ Departamento de Química Inorgánica, Facultad de Químicas, Universidad Complutense, CEI Moncloa, 28040 Madrid, Spain \\ ${ }^{3}$ Instituto de Ciencia de Materiales, CSIC, C/Sor Juana Inés de la Cruz s/n, 28049 Madrid, Spain \\ ${ }^{4}$ Instituto de Cerámica y Vidrio, CSIC, C/Kelsen 5, 28049 Madrid, Spain \\ ${ }^{5}$ ICTS Centro Nacional de Microscopía Electrónica, UCM, 28040 Madrid, Spain
}

(Received 25 July 2018; revised manuscript received 19 September 2018; published 3 January 2019)

\begin{abstract}
We describe here a type of magnetoresistance that takes place in naturally layered and outstanding ordered single phase manganites that may be mediated by magnon excitation. In particular, we show the effect for the Ruddlesden-Popper compound, $\mathrm{LaSr}_{2} \mathrm{Mn}_{2} \mathrm{O}_{7}$ synthesized by ceramic method. This material exhibits, besides the conventional colossal magnetoresistance, another type of magnetoresistance at low temperature, associated with breaking of the A-type antiferromagnetic coupling of Mn-containing planes. Excitation of magnons or application of a magnetic field breaks this antiparallel alignment so that some electrons, initially confined on the planes, become itinerant along the interplain directions through a double exchange mechanism, giving rise to resistance variations of the order of $\sim 60 \%$ for polycrystalline samples. The effect described here might be present in other types of manganites exhibiting a natural layered structure, opening up the possibility of developing magnetoresistive devices based on antiferromagnetic oxide materials without requiring artificial multilayered structures.
\end{abstract}

DOI: 10.1103/PhysRevB.99.024403

Magnetoresistance (MR) is the key property for the development of spintronic devices, such as magnetic memories or logic spin circuits. Among the different types of MR, giant magnetoresistance (GMR) [1,2], based on the relative orientation of close magnetic layers, and colossal magnetoresistance (CMR) [3], present in Mn-containing perovskites, provide MR variations large enough to be used in those devices. Nevertheless, the requirement to avoid heat dissipation by Joule effect, the improvement and miniaturization of these elements, and extending their use to the terahertz range are pushing the research of other types of magnetoresistive systems based on pure spin currents [4]. In this field, the use of magnons that may transport spin current without electrical flow [5] and antiferromagnets that do not create stray fields has led to important advances [6-8].

The Ruddlesden-Popper (RP) series, $\mathrm{A}_{n+1} \mathrm{~B}_{n} \mathrm{O}_{3 n+1}$, includes appealing materials due to the emergent phenomena that appear in low-dimensional complex oxide materials and the possibility to modify the $n$ value in order to tune their properties. Actually, the crystal structure of these phases can be described from the ordered intergrowth between $n\left(\mathrm{ABO}_{3}\right)$ perovskite $(\mathrm{P})$ blocks and one $(\mathrm{AO})$ rock-salt $(\mathrm{RS})$ layer [Fig. 1(a)], which introduces two-dimensional (2D) character as well as new possible behaviors at these interfaces. In these phases, magnetic properties are governed by the $\mathrm{BO}_{2}$ layers of the $\mathrm{P}$ unit, leading to phenomena such as MR, superconductivity, and ferroelectricity, among others [9-12]. For instance,

\footnotetext{
*luisarg@ucm.es

$\dagger$ jgcalbet@ucm.es
}

the P layer can have ferromagnetic (FM) and metallic (M) character while the RS one is insulating (I), leading to an infinite stacking of FM-M/I/FM-M layers.

Regarding the MR properties of RP series, the $n=2$ member, $\mathrm{La}_{2-2 x} \mathrm{Sr}_{1+2 x} \mathrm{Mn}_{2} \mathrm{O}_{7}(x=0.4)$, has been shown to be FM-M below $T_{\mathrm{C}}=126 \mathrm{~K}$, exhibiting large negative MR above this temperature $[13,14]$. The observed negative MR can be interpreted in a manner qualitatively similar to the case of the $n=\infty$ compound, i.e., the $\mathrm{P}$ one, as a consequence of the double exchange (DE) interaction between $\mathrm{Mn}^{3+}$ and $\mathrm{Mn}^{4+}$, leading to CMR. Nevertheless, it is worth emphasizing a substantially better low-field MR when compared with the three-dimensional (3D) perovskite. In this sense, the reduced dimensionality of the Mn-O network, due to the insertion of the RS layer, appears to dramatically enhance the MR effect $[13,14]$. Actually, the change in dimensionality can produce physical properties which strongly contrast with the 3D perovskite systems. In this case, the natural stacking of two $(\mathrm{La}, \mathrm{Sr}) \mathrm{MnO}_{3}$ perovskite layers separated by one $(\mathrm{La}, \mathrm{Sr}) \mathrm{O}$ layer forms the framework of tunneling structures and spin valves in a single chemical phase able to exhibit tunnelling MR (TMR) [14-16].

Most of the studies have been focussed on the influence of cation substitution on structures and physical properties of $\mathrm{La}_{2-2 x} \mathrm{Sr}_{1+2 x} \mathrm{Mn}_{2} \mathrm{O}_{7}$ compounds [17], leading to the conclusion that their behavior is very sensitive to the lanthanide ( $\mathrm{Ln})$ type, $\mathrm{Ln} / \mathrm{Sr}$ ratio and ordering degree $[12,18,19]$. Regarding this point, notice the difficulty to stabilize pure highordered terms using chemical methods and its limitation to develop new functionalities in this family. In this sense, Battle et al. [19] have previously reported that attempts to 


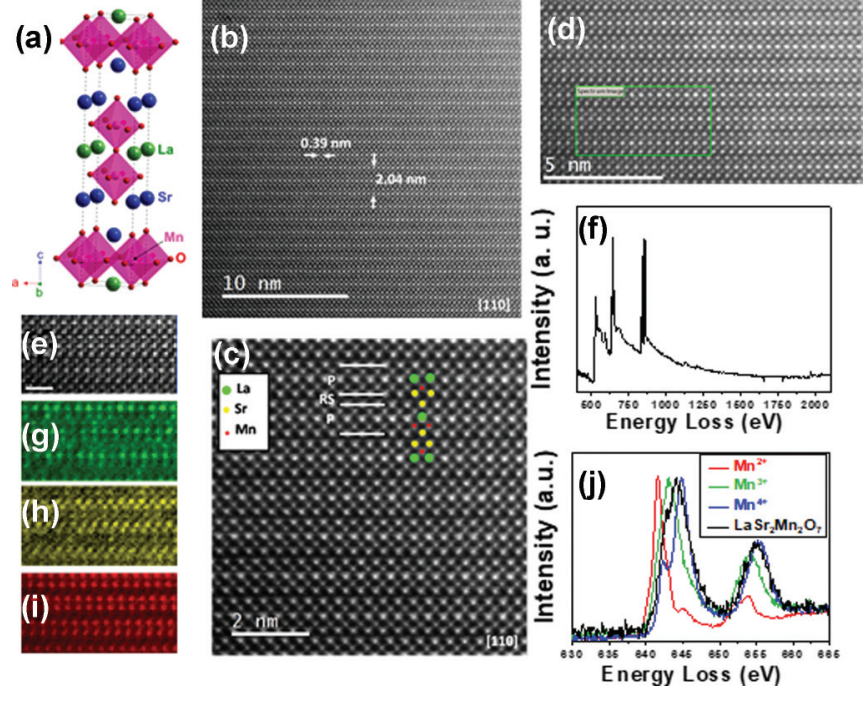

FIG. 1. (a) Structural model corresponding to the $n=2$ RP term $\mathrm{A}_{3} \mathrm{~B}_{2} \mathrm{O}_{7}$ (La = green, $\mathrm{Sr}=$ blue, $\mathrm{Mn}=$ pink, $\mathrm{O}=$ red). (b) HAADF image along [110̄]. (c) Enhanced detail of (b) showing the atomically resolved $\mathrm{La}, \mathrm{Sr}$, and Mn columns. (d) HAADF image of the selected area for spectrum image analysis. (e) HAADF image of the region of interest recorded at the same time as the EELS data set acquisition. (f) EELS spectral sum acquired over the area marked in (d), showing the $\mathrm{La}-\mathrm{M}_{4,5}, \mathrm{Sr}-\mathrm{L}_{2,3}, \mathrm{Mn}-\mathrm{L}_{2,3}$, and $\mathrm{O}-\mathrm{K}$ edges. Chemical

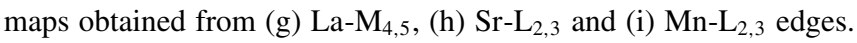
(j) $\mathrm{Mn}-\mathrm{L}_{2,3}$ edge of the sample in comparison with reference samples containing $\mathrm{Mn}^{2+}, \mathrm{Mn}^{3+}$, and $\mathrm{Mn}^{4+}$.

prepare $\mathrm{LaSr}_{2} \mathrm{Mn}_{2} \mathrm{O}_{7}$ lead to, at least, two different chemical phases: an antiferromagnetic (AFM) majority one (81\%), $\mathrm{Sr}_{2.04} \mathrm{La}_{0.96} \mathrm{Mn}_{2} \mathrm{O}_{7}\left(T_{N} \sim 211 \mathrm{~K}\right)$, and a FM minority one with the $\mathrm{Sr}_{1.8} \mathrm{La}_{1.2} \mathrm{Mn}_{2} \mathrm{O}_{7}$ composition $\left(T_{\mathrm{C}} \sim 126 \mathrm{~K}\right)$. Even more, in most cases, disordered intergrowths between the basic unities have been reported [19]. As a consequence, their electric and magnetic behavior is modified leading to an apparent discrepancy in the properties of both polycrystalline materials and thin films. The presence of intergrowths has also been shown in thin films prepared by molecular-beam epitaxy [12,20,21], requiring the use of sophisticated characterization techniques, such as synchrotron x-ray scattering and high resolution scanning transmission electron microscopy, to ascertain the achievement of defect-free thin films. Nevertheless, we have recently shown [22] how using the ceramic method, with a very slow cooling process, it is possible to stabilize bulk $\mathrm{La}_{0.5} \mathrm{Ca}_{2.5} \mathrm{Mn}_{2} \mathrm{O}_{7}$ as crystalline particles of several microns in size without extended defects or disordered intergrowths. In this sense, the control of defects and ordered cation distribution at atomic scale in the layers constituting RP phases could promote the appearance of emerging phenomena and tuning of the material properties.

In order to shed light on the complex behavior of these RP systems and to distinguish intrinsic effects from those due to the presence of defects, we focused on the system $\mathrm{La}_{2-2 x} \mathrm{Sr}_{1+2 x} \mathrm{Mn}_{2} \mathrm{O}_{7}$, which displays interesting features near half doping $(x=0.5)$, where long-range orbitals and CE-type orbitals and charge order (CO) are predicted [23]. For that composition, it has been commonly accepted that the $\mathrm{CO}$ state is reentrant. It appears below $\sim 220 \mathrm{~K}$. With further decreasing temperature, the $\mathrm{CO}$ state diminishes and the A-type AFM phase appears at $170 \mathrm{~K}$. Finally, the CO state collapses below 50-100 K [17,24-28]. In this bilayer manganite, coexistence of CE order and A-type AFM, between 100 and $200 \mathrm{~K}$, is also universally reported. It is worth recalling that in an A-type AFM structure Mn atoms are FM coupled within $\mathrm{P}$ layers parallel to the $a b$ plane, these layers being AFM coupled along the $c$ direction. Consequently, the conductivity on this type of materials is highly anisotropic: the system allows electrical conduction within the Mn-containing planes but it is an insulator in the perpendicular direction. In this bilayered manganite, coexistence of $\mathrm{CE}$ order and A-type AFM between $\sim 100$ and $\sim 220 \mathrm{~K}$ is universally reported [17,24-28]. However, at low temperature, most of the authors describe only an A-type AMF phase [29] whereas for a minority the CE-type order is the low-temperature ground state and reentry only occurs for small inhomogeneities in cation or oxygen composition [26]. The coexistence of CEtype order and A-type AFM at low temperature has also been described [30]. According to these controversial results, the motivation of this paper is based on the stabilization of a wellordered $\mathrm{LaSr}_{2} \mathrm{Mn}_{2} \mathrm{O}_{7}$ material following an alternative and simple synthesis pathway. Since physical properties are very sensitive to both elemental composition and subtle structural changes, a comprehensive understanding of these features requires precise chemical and crystallographic knowledge. In this sense, $\mathrm{LaSr}_{2} \mathrm{Mn}_{2} \mathrm{O}_{7}$ crystalline particles have been chemically and structurally characterized for the first time at the atomic level using aberration corrected electron microscopy. Once the single and ordered phase is verified by diffraction techniques and atomic resolution electron microscopy, the peculiar electric behavior is reported in order to provide a reasonable explanation of the dramatic thermal increase of the conductivity at low temperatures.

We here describe a MR effect in RP series associated with the control of current flow between Mn-containing planes AFM coupled. The MR can be mediated by thermal excitation of magnons that breaks the antiparallel alignment of Mn-containing layers allowing electrical conduction by DE mechanism.

In order to shed light on the complex behavior of this compound and to separate intrinsic effects from those due to the presence of defects, $\mathrm{LaSr}_{2} \mathrm{Mn}_{2} \mathrm{O}_{7}$ micrometric particles were synthesized by ceramic method with an ultraslow quenching method [31]. The sample consists of monocrystalline micrometric particles the average composition of which is in agreement with the nominal one [32]. Neutron-diffraction (ND) study [33] of $\mathrm{LaSr}_{2} \mathrm{Mn}_{2} \mathrm{O}_{7}$, at room temperature, confirms the stabilization of a single phase which can be indexed on the basis of a tetragonal cell (SG $I 4 / \mathrm{mmm}$ ) and lattice parameters $a=3.8730(1)$ and $c=19.995(1) \AA[34,35]$. In order to obtain a more comprehensive view of the structural and compositional features, related to short-range orderdisorder phenomena, an exhaustive characterization at atomic resolution, using high angle annular dark field (HAADF) imaging and electron energy-loss spectroscopy (EELS), was performed (Fig. 1) [36]

The HAADF image in Fig. 1(b) shows a well-ordered distribution of atomic columns with periodicities of 0.39 and 
$2.04 \mathrm{~nm}$, characteristic of a $n=2 \mathrm{RP}$ term, in agreement to ND. Besides this information, the image provides compositional information since the contrast of atomic columns is related to the atomic number $(Z)$. The brightest contrast corresponds to the heavier cations, i.e., $\mathrm{La}(Z=57)$ and $\operatorname{Sr}(Z=38)$, while the less bright is due to $\operatorname{Mn}(Z=25)$. Furthermore, the HAADF image clearly reflects that the A sites $\left(\mathrm{A}_{n+1} \mathrm{~B}_{n} \mathrm{O}_{3 n+1}\right)$, corresponding to the $\mathrm{P}\left(\mathrm{ABO}_{3}\right)$ and $\mathrm{RS}$ (AO) unities, exhibit different brightness. This must be related to different A site occupation, i.e., $\mathrm{La}$ and Sr. According to Z, $\mathrm{La}$ and $\mathrm{Sr}$ would be preferentially distributed over the central site of $\mathrm{P}$ and RS blocks, respectively, as represented in the enhanced image detail in Fig. 1(c). In order to confirm this cationic distribution, an atomically resolved EELS study was performed [Figs. 1(d)-1(i)]. The HAADF image recorded simultaneously to EELS acquisition and the corresponding sum spectra are depicted in Figs. 1(e) and 1(f), respectively. The resulting chemical maps, for each individual signal, are shown in Figs. 1(g)-1(i), indicating that $\mathrm{Mn}$ is always located at the $\mathrm{B}$ sites of the $\mathrm{P}$ lattice, $\mathrm{La}$ at the A sites of $\mathrm{P}$ blocks, and $\mathrm{Sr}$ at the A sites of the RS layers. This well-ordered distribution, both in terms of crystallography and composition, is probably due to the very slow cooling process performed as previously described in the synthesis of the related $\mathrm{La}_{0.5} \mathrm{Ca}_{2.5} \mathrm{Mn}_{2} \mathrm{O}_{7}$ compound [22].

On the other hand, $\mathrm{Mn}^{3+}$ and $\mathrm{Mn}^{4+}$ oxidation states [36] coexist in agreement with the nominal composition (50\% $\mathrm{Mn}^{4+}$ and $50 \% \mathrm{Mn}^{3+}$ ). Figure 1(j) shows a representative $\mathrm{Mn}-\mathrm{L}_{2,3}$ signal of the $\mathrm{LaSr}_{2} \mathrm{Mn}_{2} \mathrm{O}_{7}$ sample, compared to the standards for $\mathrm{Mn}^{4+}\left(\mathrm{Ca}_{2} \mathrm{Mn}_{3} \mathrm{O}_{8}\right), \mathrm{Mn}^{3+}\left(\mathrm{LaMnO}_{3}\right)$, and $\mathrm{Mn}^{2+}\left(\mathrm{CaMnO}_{2}\right)$. Details of the synthesis conditions of the standards are described in [37-39]. The relative intensity of the $\mathrm{Mn}-\mathrm{L}_{2}$ and $\mathrm{Mn}-\mathrm{L}_{3}$ white lines also supports the presence of both oxidation states [36]. For completeness, the oxygen sublattice has also been visualized using annular bright field (ABF) technique [36], being in agreement to the characteristic distribution of the oxygen atoms at the octahedral sites in RP $n=2$.

The magnetic and electric characterization of the sample is summarized in Fig. 2. Measurements are in agreement with previous reported data [24-30,40-43]. However, as far as we know, no explanation concerning the peculiar electric behavior at low temperature has been reported. Electrical resistivity [Fig. 2(a)] exhibits an initial decrease as the temperature increases from 5 to $60 \mathrm{~K}$. Subsequently, the resistivity increases reaching a maximum at about $160 \mathrm{~K}$, and then decreases down to almost zero at room temperature. The resistivity in presence of an 8-T magnetic field presents the same trend but with reduced values of resistivity in the whole range of temperatures. MR shown in Fig. 2(b) decreases with temperature, exhibiting a plateau between 80 and $120 \mathrm{~K}$ and a sharp peak at about $230 \mathrm{~K}$. Magnetization measured in field cooled (FC) and zero-field cooled (ZFC) conditions [Fig. 2(c)] presents a weak dependence on temperature up to $160 \mathrm{~K}$, where both curves collapse and drop to zero at $300 \mathrm{~K}$. The magnetization curve at $5 \mathrm{~K}$ [Fig. 2(c) inset] exhibits a hysteresis loop that saturates at about 1-T field, plus a linear component. Finally, Fig. 2(d) presents the dependence of the MR with the magnetic field, which is maximum at zero field and tends to become a cusp point as the temperature decreases. A remarkable value of
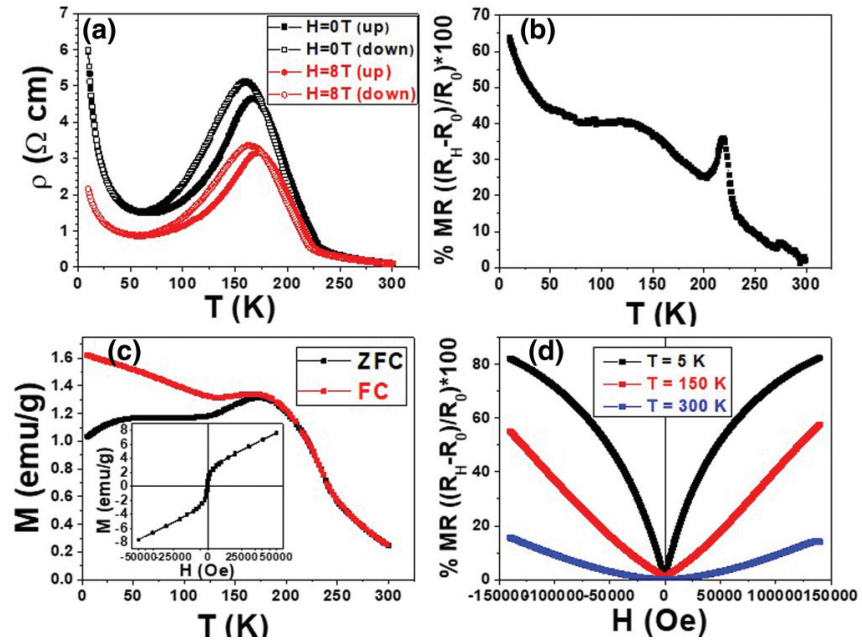

FIG. 2. (a) Resistivity obtained on heating (up) and cooling (down) in zero field and under a field of $8 \mathrm{~T}$. (b) Magnetoresistance under $8 \mathrm{~T}$. (c) Magnetization in zero field and field conditions as a function of temperature under 1000 Oe. Inset: Field dependence of the magnetization at $5 \mathrm{~K}$. (d) MR measurements as a function of applied magnetic field at 5, 150, and $300 \mathrm{~K}$.

$82 \%$ of MR is obtained at $5 \mathrm{~K}$. Additionally, the magnetic structure at low temperature $(3 \mathrm{~K})$ has been determined by ND study, showing an A-type AFM phase [44] in agreement with previous reported works $[19,20]$. This result is apparently contradictory with the observation of a hysteresis loop at $5 \mathrm{~K}$ shown in the inset in Fig. 2(c).

The origin of the electric and magnetic behavior that we found in the range 60 to $300 \mathrm{~K}$ is similar to that previously described in the literature for other Mn related layered perovskites. According to that, the simultaneous coexistence of FM, AFM, and CO correlations determines the transport and magnetotransport properties.

The increase of resistivity above $60 \mathrm{~K}$ [Fig. 2(a)] corresponds to the development of a $\mathrm{CO}$ state, leading to a maximum of resistivity at $160 \mathrm{~K}[30,40]$. The subsequent decrease of resistivity when increasing temperature reflects the disappearance of the $\mathrm{CO}$ state, whereas the local maximum of $\mathrm{MR}$ at $230 \mathrm{~K}$ indicates the signature of typical CMR, pointing out that some FM order within the perovskite planes remains up to this temperature. Therefore, it turns out that $230 \mathrm{~K}$ is the Curie temperature corresponding to the interplane $\mathrm{DE}$ coupling [28] as confirmed by the collapse of the ZFC-FC curves in Fig. 2(c).

Although the significant decrease of resistivity and MR in the range 5 to $60 \mathrm{~K}$ has been observed in samples prepared with different techniques, its origin has not been yet established [30,41-43]. The fact that these features have been observed in a sample with outstanding crystallographic and compositional order, achieved with the ultralow cooling process, points out that it is not due to segregation of secondary phases nor disorder but intrinsic of the material.

The observed dependence of resistivity with temperature in the range 5-60 $\mathrm{K}$ is opposite to that typical of metals for which resistivity increases with the temperature [44]. In contrast, a decrease of resistivity with temperature is found 
in semiconductors and similar systems where the increase of temperature generates carriers [45]. In those materials, increasing the temperature provides the thermal energy to delocalized charges, increasing in this way the number of carriers and consequently reducing the resistivity. However, for this mechanism, the presence of a magnetic field is irrelevant while we observe that for our system the application of a magnetic field produces also a significant decrease of resistivity discarding this carrier-generation mechanism. In particular, as shown in Fig. 2(a), application of an 8-T field has the same effect as increasing the temperature up to $25 \mathrm{~K}$. Since the effect of the field is to promote DE conductivity by breaking the perfect AFM order, it can be inferred that a similar mechanism appears by increasing the temperature. As is well known, magnons are collective deviations of the spin orientations at each AFM sublattice that are thermally excited [45]. Therefore, the effect of heating, in a similar fashion to the applied field, may be to destroy the perfect AFM, giving rise to the possibility of $\mathrm{DE}$ electron jumping.

As thoroughly described, $\mathrm{LaSr}_{2} \mathrm{Mn}_{2} \mathrm{O}_{7}$ corresponds to RP series with two $\mathrm{P}$ sheets separated by a RS layer. At low temperature, the $\mathrm{P}$ planes are internally FM coupled and exhibit electrical conductivity within the plane. However, adjacent $P$ layers are AFM coupled, leading to large electrical resistivity. Thus, for polycrystalline samples with randomly oriented particles (as those studied here), the electrical resistivity will arise mainly from those regions in which the electrical current must flow perpendicular to the P layers [46]. Consequently, the large variations of resistivity that are observed upon temperature increase or application of a magnetic field must be related to the changes in conductivity between those layers AFM coupled.

In the case of $\mathrm{Mn}$ related perovskites, the effect of the magnetic field in electrical conductance is well known. The electrical conduction in these FM materials takes place by DE between $\mathrm{Mn}^{3+}$ and $\mathrm{Mn}^{4+}$ atoms with parallel magnetic moment and separated by $\mathrm{O}$ atoms. This is described by the Zener model $[47,48]$ for which the conductivity is given by

$$
\sigma=\frac{x e^{2} t_{i j} \cos \left(\frac{\alpha}{2}\right)}{a h K_{B} T}
$$

where $x$ is the concentration of $\mathrm{Mn}^{4+}$ available for hopping, $t_{i j}$ is the hopping integral, $\alpha$ is the angle between the magnetic moment of the $\mathrm{Mn}^{3+}$ and $\mathrm{Mn}^{4+}$ atoms in adjacent planes, and $a$ is the lattice constant. It should be noted that $t_{i j}$ is the average hopping integral corresponding to tunneling between adjacent planes and tunneling through the RS-like barrier.

In absence of magnetic field, the AFM alignment between Mn atoms $(\alpha=\pi)$ of adjacent planes prevents electrical conduction $(\sigma=0)$ as shown in Fig. 3. Upon application of a magnetic field, the magnetic moments of $\mathrm{Mn}$ atoms tend to align with the field in order to reduce the Zeeman energy, thus breaking their perfect antiparallelism and allowing some electrical conduction $(\sigma \neq 0)$. Increasing the temperature promotes the appearance of magnons that will propagate in all directions, including that perpendicular to the AFM planes. These magnons break the antiparallel orientation of the magnetic moments of Mn atoms from the AFM planes as Fig. 3 shows. In this situation $(\alpha \neq \pi)$ and, according to
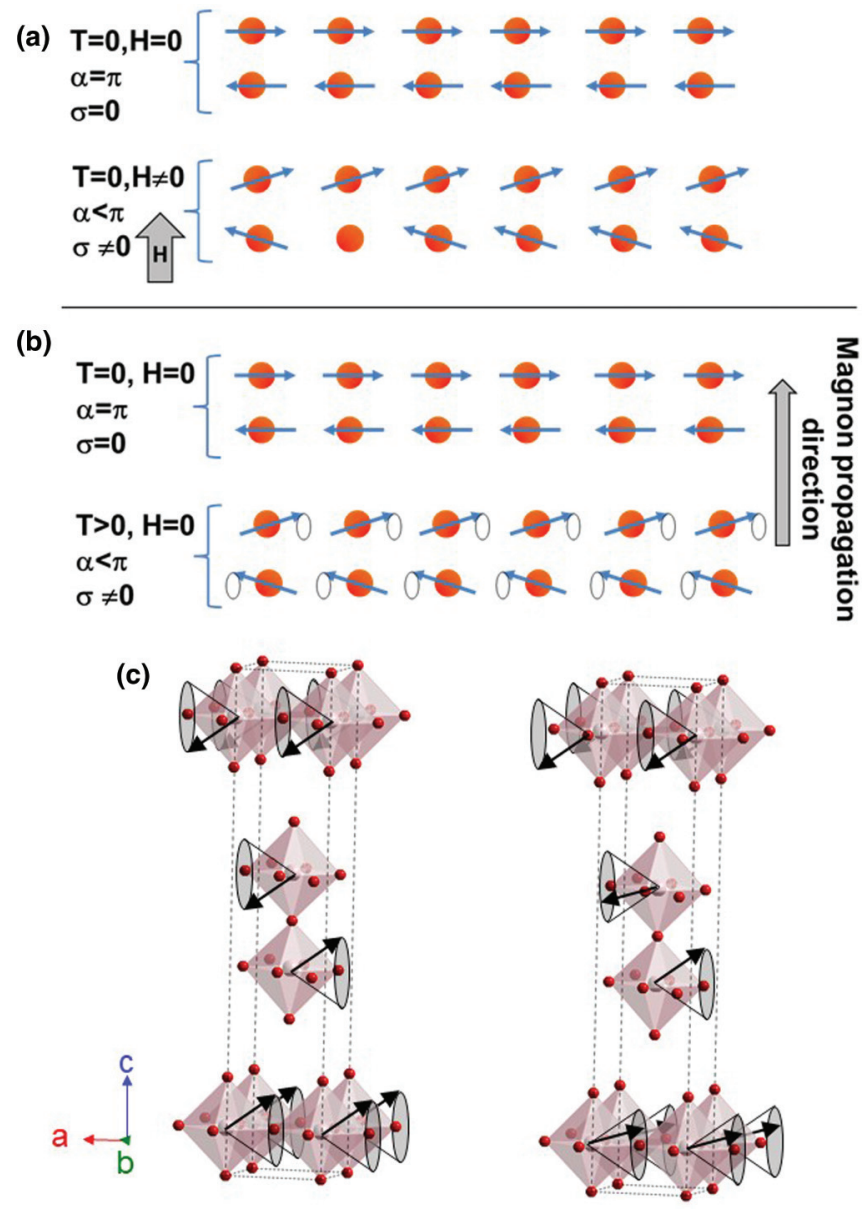

FIG. 3. Model layout of thermal and magnetic dependence of resistivity. At $0 \mathrm{~K}$ and in absence of magnetic field, the Mn atoms of adjacent planes are antiparallel $(\alpha=\pi)$. (a) Application of a magnetic field tends to align the magnetic moment of $\mathrm{Mn}$ atoms with $\mathrm{H}$ reducing their relative angles and allowing electrical conduction. (b) Increasing the temperature generates magnons that yield a dephasing between magnetic moments of adjacent planes, allowing electrical conduction. (c) Schematic representation of Mn magnetic moment corresponding to $T=0, H=0$ (left) and $T \neq 0$ (or $H \neq 0$ ) (right), inserted in RP structure.

Eq. (1), some electrical conduction will be permitted $(\sigma \neq 0)$. As the temperature increases, the density of magnons also does, leading to smaller values of the angle between magnetic moments of $\mathrm{Mn}$ atoms, and therefore reducing the resistivity as experimentally observed.

The observation of this effect requires an outstanding order of cation distribution as that achieved with the ultralow quenching method. Segregation of $\mathrm{La}$ or $\mathrm{Sr}$ would induce clustering of $\mathrm{Mn}^{3+} / \mathrm{Mn}^{4+}$ obstructing conduction via double exchange [22].

The proposed mechanism is, in some sense, equivalent to GMR, as the conductivity depends on the relative orientation of magnetization of two magnetic regions [1,2]. However, in our case, the regions are two adjacent $\mathrm{MnO}_{2}$ perovskite monolayers confined by RS layers without a physical barrier and no reversal of magnetization in one of the layers is required. This particularity means that the observed MR can be 


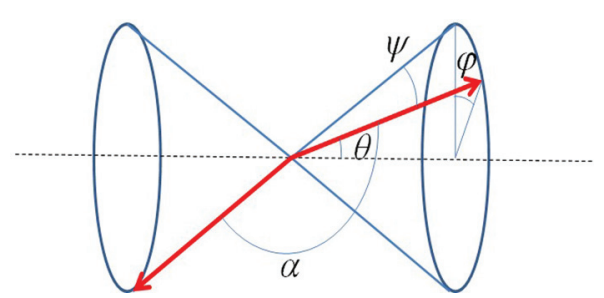

FIG. 4. Diagram of magnetic moments (red arrows), AFM aligned, precessing with angle $\theta$ and with dephasing $\varphi$ between them. For clarity, both magnetic moments are placed at the origin.

also thermally excited: the magnons break the antiparallelism between the planes, allowing some conduction.

In presence of magnons propagating along the direction perpendicular to two Mn atoms in adjacent planes AFM coupled, both magnetic moments will be precessing as illustrated in Fig. 4. The excitation of magnons leads to a precession of the magnetic moments with an angle $\theta$ with respect to the ground AFM state, and the wave vector of the magnon induces a dephasing between the magnetic moments of adjacent $\mathrm{Mn}$ of an angle $\varphi$. The angle $\theta$ is proportional to the root square of the density of 2D FM magnons excited in the $a b$ planes. For a 2D system, the density of FM magnons is proportional to the temperature [49] and, consequently, $\theta$ is proportional to $\mathrm{T}^{1 / 2}$.

Then, the angle between the magnetic moments is

$$
\alpha=\pi-\psi=\pi-\theta \cdot \varphi .
$$

The angle $\varphi$ corresponds to the dephasing between magnetic moments of adjacent $\mathrm{Mn}$ atoms separated along the $c$ axis, induced by excitation of AFM magnons. This angle is also proportional to the root square of the number of magnons. For a one-dimensional antiferromagnet the number of magnons is proportional to $T$ and, thereby, $\varphi \propto T^{1 / 2}$ [49].

Thus, for small magnetic excitations (i.e., the lowtemperature regime) the component of the hopping transfer integral between $i$ and $j$ ions is given by

$t_{i j} \cos (\alpha / 2)=t_{i j} \cos \left(\frac{\pi-\theta \cdot \varphi}{2}\right)=t_{i j} \sin \left(\frac{\theta \cdot \varphi}{2}\right) \approx t_{i j} \gamma T$

$\gamma$ being a constant. The transfer integral should correspond to the average corresponding to $\mathrm{P}$ and RS layers.

However, in our case, the AFM order corresponding to the ground state renders impossible any jump. It is the temperature itself by means of the magnons that promotes the availability. Therefore $x$ should be substituted by $x \cos (\alpha / 2)$ :

$$
\sigma=\frac{x e^{2} t_{i j}\left(\cos \left(\frac{\alpha}{2}\right)\right)^{2}}{a h K_{B} T}=\frac{x e^{2} t_{i j} \gamma^{2} T^{2}}{a h K_{B} T}=\frac{x e^{2} t_{i j} \gamma^{2} T}{a h K_{B}} .
$$

Hence, for the low-temperature range, the linear dependence of the conductivity $\sigma$ with temperature given by [5] yields for the resistivity the following dependence:

$$
\rho=\frac{\rho_{0}}{T} \text {. }
$$

The resistivity within the Mn-containing plane will depend weakly on the temperature in this range, and therefore for

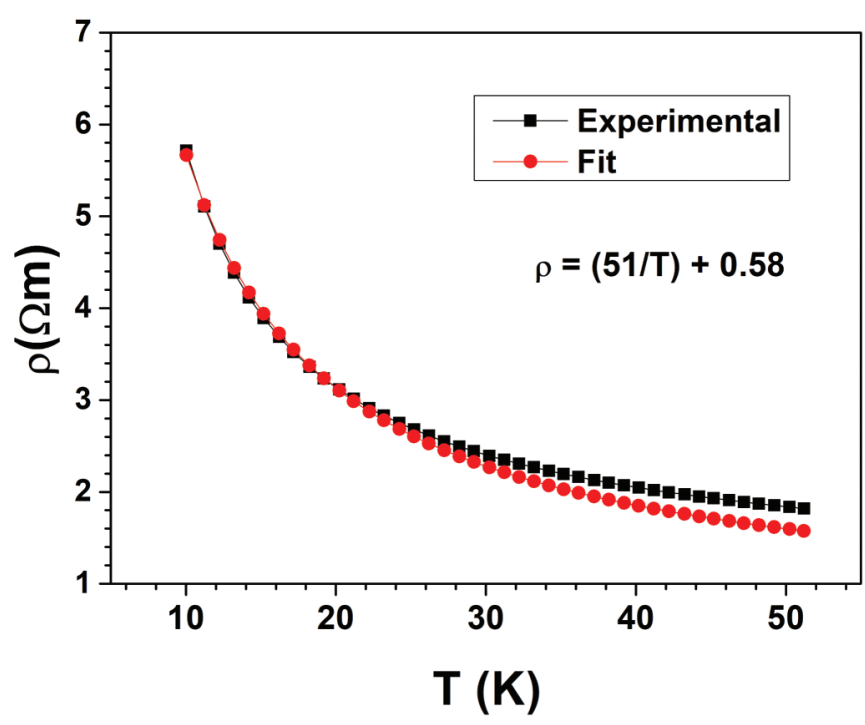

FIG. 5. Experimental thermal dependence of the electrical resistivity in the range 5 to $50 \mathrm{~K}$ and fit to Eq. (6) with the parameters indicated in the figure.

polycrystalline samples the total resistivity will be given by

$$
\rho=\frac{\rho_{0}}{T}+C
$$

Figure 5 shows the experimental dependence of the resistivity with temperature in the range 5 to $50 \mathrm{~K}$ and the fit to Eq. (6). This behavior is representative of all samples analyzed in this paper: the evolution of the other four samples is identical to the one shown here and is not included in Fig. 5 for clarity. As it can be seen, there is a good match between both curves up to $25 \mathrm{~K}$, where, according to Fig. 2(a), the onset $\mathrm{CO}$ state gives rise to a different thermal behavior. This model allows also explaining the apparent discrepancy between the ND studies at $3 \mathrm{~K}$ with the observation of a hysteresis loop measured at $5 \mathrm{~K}$. In absence of applied magnetic field and at low temperature (3-5 K), the system exhibits AFM detected by ND. However, the weak AFM exchange between planes in the A-type AFM can be overcome by a moderate field of the order of $1 \mathrm{~T}$ (or by a temperature increase), leading to the observation of a hysteresis loop due to in-plane FM of the A-type AFM phase.

When a magnetic field is applied along the $c$ axis, the Mn spins would rotate from the initial rotation following the relationship

$$
\cos (\alpha / 2)=\frac{\mu_{0} M_{S}}{2 K} H=\chi H
$$

where $K$ is the magnetic anisotropy and $M_{s}$ is the saturation magnetization of the FM $a b$ planes and $H$ is the average value of the applied field along the $c$ axis (which for a polycrystalline sample with random orientation is $0.5 H_{\text {applied }}$ ). Therefore, for a small variation of $T$ the transfer integral between $i$ and $j$ ions becomes

$$
t_{i j} \cos (\alpha / 2)=t_{i j}[a T+\chi(T) H] .
$$


Hence, the Zener conductivity

$$
\begin{aligned}
\sigma & =\frac{x e^{2} t_{i j}\left[\cos \left(\frac{\alpha}{2}\right)\right]^{2}}{a h K_{B} T}=\frac{x e^{2} t_{i j}[a T+\chi(T) H]^{2}}{a h K_{B} T} \\
& =\frac{x e^{2} t_{i j}[a+m(T) H] T}{a h K_{B}}
\end{aligned}
$$

where

$$
m(T)=\frac{\chi(T)}{T} .
$$

Then, the resistivity can be expressed as

$$
\rho=\frac{\rho_{0}}{T+b(T) H} .
$$

According to this equation, resistivity diverges to infinite as $T, H \rightarrow 0$. This is in agreement with the experimental results shown in Fig. 2(d): for low temperatures, the MR curve shows a cusp point at $H=0$.

Further quantitative confirmation of the proposed model can be obtained by comparing the energy provided by the temperature and magnetic field and their effect on the electrical resistance. From Fig. 2(a), at $5 \mathrm{~K}$, the application of an 8-T magnetic field yields a reduction of the electric resistivity from 6 to $2.1 \Omega \mathrm{cm}$. The same reduction of $65 \%$ is achieved increasing the temperature $\sim 20 \mathrm{~K}$. The average magnetic moment per $\mathrm{Mn}$ atom for a mixture of $50 \% \mathrm{Mn}^{3+}$ and $50 \%$ $\mathrm{Mn}^{4+}$ is $3.5 \mu_{\mathrm{B}}$. Hence, application of an 8-T field provides a Zeeman energy of $E_{\mathrm{Z}}=3.5 \mu_{\mathrm{B}}, B \sim 1.62 \mathrm{meV}$. Similarly, increasing the temperature $20 \mathrm{~K}$ provides thermal energy of about $K_{B} T \sim 1.72 \mathrm{meV}$. This estimation points out that for the same energy provided by thermal excitation or application of magnetic field the reduction of the resistivity is of the same order, confirming that, in both cases, the mechanism provides the energy required to overcome the AFM coupling between adjacent planes, allowing then electrical conduction by DE mechanism.
The conductivity in these RP compounds at low temperature can be hence described as similar to GMR between Mn containing planes. When both planes are AFM coupled, they are in a high resistance state. Application of a magnetic field or increasing the temperature induces some parallelism, allowing electrical conduction as it happens in GMR. However, while conventional GMR is independent of temperature (provided that the material is well below the $T_{\mathrm{C}}$ of the ferromagnets) in the model described here, the GMR can be mediated via temperature. Note that, in order to observe this effect, the layered structure with A-type AFM is required, so that the resistivity is very sensitive to interplanar coupling. In fact, $\mathrm{La}_{1.2} \mathrm{Sr}_{1.8} \mathrm{Mn}_{2} \mathrm{O}_{7}$, that belongs also to the RP series with $n=2$ but without A-type AFM [17], does not exhibit the low-temperature increase of resistance nor MR [50].

In summary, we found here that the $\mathrm{LaSr}_{2} \mathrm{Mn}_{2} \mathrm{O}_{7} \mathrm{RP}$ phase, exhibiting CMR close to its Curie temperature, shows additional MR at low temperature associated with its layered structure. This MR corresponds to a rupture of the perfect AFM coupling of magnetic moments of Mn atoms in adjacent planes that can be induced by applying a magnetic field or excitation of magnons, so both thermal and magnetic tuning of the resistance are possible. It is noteworthy that excitation of magnons in $\mathrm{MnO}_{2} \mathrm{P}$ monolayers separated by RS layers reduces electrical resistivity, a behavior opposite to that previously described for other types of magnetic materials. Consequently, this represents a mechanism for the control of resistance via magnons. This control could be exerted by any local heat source such as electrical currents or optical excitation, which could be applied for the development of new magnonic devices.

This work was supported by the Spanish Ministry of Innovation, Science, and Technology and the Spanish Ministry of Economy and Competitiveness through Research Projects No. CSD2009-00013, No. TSI-020100-2011-280, No. MAT201123068, No. MAT2012-37109-C02-01, No. MAT2014-54372$\mathrm{R}$, and No. MAT2017-86450-C04-R.

All authors have given approval to the final version of the paper. All authors contributed equally.
[1] M. N. Baibich, J. M. Broto, A. Fert, F. Nguyen Van Dau, F. Petroff, P. Etienne, G. Creuzet, A. Friederich, and J. Chazelas, Phys. Rev. Lett. 61, 2472 (1988).

[2] G. Binasch, P. Grünberg, F. Saurenbach, and W. Zinn, Phys. Rev. B 39, 4828 (1989).

[3] A. J. Millis, Theory of CMR Manganites in Colossal Magnetoresistance Oxides: Advances in Condensed Matter Science, edited by Y. Tokura (Gordon and Breach, New York, 2000).

[4] T. Jungwirth, J. Wunderlich, and K. Olejník, Nat. Mater. 11, 382 (2012).

[5] A. V. Chumak, V. I. Vasyuchka, A. A. Serga, and B. Hillebrands, Nat. Phys. 11, 453 (2015).

[6] H. Wu, L. Huang, C. Fang, B. S. Yang, C. H. Wan, G. Q. Yu, J. F. Feng, H. X. Wei, and X. F. Han, Phys. Rev. Lett. 120, 097205 (2018).
[7] Z. Qiu, D. Hou, J. Barker, K- Yamamoto, O. Gomonay, and E. Saitoh, Nat. Mater. 17, 577 (2018).

[8] T. Jungwirth, X. Marti, P. Wadley, and J. Wunderlich, Nat. Nanotech. 11, 231 (2016).

[9] F. Masse, S. De Jong, Y. Huang, W. K. Siu, I. Santoso, A. Mans, A. T. Boothroyd, D. Prabhakaran, R. Follath, A. Varykhalov, L. Patthey, M. Shi, J. B. Goedkoop, and M. S. Golden, Nature Physics, 7, 978 (2011).

[10] A. T. Mulder, N. A. Benedek, J. M. Rondinelli, and C. J. Fennie, Adv. Funct. Mater. 23, 4810 (2013).

[11] C. H. Lee, N. D. Orloff, T. Birol, Y. Zhu, V. Goian, E. Rocas, R. Haislmaier, E. Vlahos, J. A. Mundy, L. F. Kourkoutis, Y. Nie, M. D. Biegalski, J. Zhang, M. Bernhagen, N. A. Benedek, Y. Kim, J. D. Brock, R. L. Uecker, X. X. Xi, V. Gopalan, D. Nuzhnyy, S. Kamba, A. Muller, I. Takeuchi, J. C. Booth, C. J. Fennie, and D. G. Schlom, Nature (London) 502, 532 (2013). 
[12] G. Rijnders, Nat. Mater. 13, 844 (2014).

[13] Y. Moritomo, A. Asamitsu, H. Kuwahara, and Y. Tokura, Nature (London) 380, 141 (1996).

[14] T. Kimura, Y. Tomioka, H. Kuwahara, A. Asamitsu, M. Tamura, and Y. Tokura, Science 274, 1698 (1996).

[15] T. G. Perring, G. Aeppli, T. Kimura, Y. Tokura, and M. A. Adams, Phys. Rev. B 58, R14693 (1998).

[16] T. Kimura, A. Asamitsu, Y. Tomioka, and Y. Tokura, Phys. Rev. Lett. 79, 3720 (1997).

[17] C. D. Ling, J. E. Millburn, F. Mitchell, D. N. Argyriou, J. Linton, and H. N. Bordallo, Phys. Rev. B 62, 15096 (2000).

[18] P. D. Battle, M. A. Green, N. S. Laskey, J. E. Millburn, L. Murphy, M. J. Rosseinsky, S. P. Sullivan, and J. F. Vente, Chem. Mater. 9, 552 (1997).

[19] P. D. Battle, D. E. Cox, M. A. Green, J. E. Millburn, L. E. Spring, P. G. Radaelli, M. J Rosseinsky, and J. F. Vente, Chem. Mater. 9, 1042 (1997).

[20] J. H. Lee, G. Luo, I. C. Tung, S. H. Chang, Z. Luo, M. Malshe, M. Gadre, A. Bhattacharya, S. M. Nakhmanson, J. A. Eastman, H. Hong, J. Jellinek, D. Morgan, D. Fong, and J. W. Freeland, Nature Mater. 13, 879 (2014).

[21] N. F. Yie, Y. Zhu, C.-H. Lee, L. F. Kourkoutis, J. A. Mundy, J. Junquera, Ph. Ghosez, D. J. Baek, S. Sung, X. X. Xi, K. M. Shen, D. A. Muller, and D. G. Schlom, Nature Comm. 5, 4530 (2014).

[22] L. Ruiz-González, D. González-Merchante, R. Cortés-Gil, J. M. Alonso, J. L. Martínez, A. Hernando, and J. M. GonzálezCalbet, Chem. Mater. 27, 1397 (2015).

[23] J. B. Goodenough, Phys. Rev. 100, 564 (1955).

[24] T. Kimura, R. Kumai, Y. Tokura, J. Q. Li, and Y. Matsui, Phys. Rev. B 58, 11081 (1998).

[25] T. Chatterji, G. J. McIntyre, W. Caliebe, R. Suryanarayanan, G. Dhalenne, and A. Revcolevschi, Phys. Rev. B 61, 570 (2000).

[26] Qing'An. Li, K. E. Gray, H. Zheng, H. Claus, S. Rosenkranz, S. Nyborg Ancona, R. Osborn, J. F. Mitchell, Y. Chen, and J. W. Lynn, Phys. Rev. Lett. 98, 167201 (2007).

[27] J. E. Medvedeva, V. I. Anisimov, M. A. Korotin, O. N. Ryasov, and A. J. Freeman, J. Magn. Magn. Mater. 237, 47 (2001).

[28] Q. Zhang, W. Zhang, and Z. Jiang, Phys. Rev. B 72, 142401 (2005).

[29] D. N. Argyriou, H. N. Bordallo, B. J. Campbell, A. K. Cheetham, D. E. Cox, J. S. Gardner, K. Hanif, A. dos Santos, and G. F. Strouse, Phys. Rev. B 61, 15269 (2000).

[30] J. S. Lee, C. C. Kao, C. S. Nelson, H. Jang, K. T Ko, S. B. Kim, Y. J. Choi, S. W. Cheong, S. Smadici, P. Abbamonte, and J. H Park, Phys. Rev. Lett. 107, 037206 (2011).
[31] See Supplemental Material at http://link.aps.org/supplemental/ 10.1103/PhysRevB.99.024403 for details of experimental synthesis conditions.

[32] See Supplemental Material at http://link.aps.org/supplemental/ 10.1103/PhysRevB.99.024403 for details of low magnification TEM and average compositional analysis.

[33] See Supplemental Material at http://link.aps.org/supplemental/ 10.1103/PhysRevB.99.024403 for details of neutron-diffraction characterization at room temperature.

[34] S. N. Ruddlesden and P. Popper, Acta Crystallogr. 10, 538 (1957).

[35] S. N. Ruddlesden and P. Popper, Acta Crystallogr. 11, 54 (1958).

[36] See Supplemental Material at http://link.aps.org/supplemental/ 10.1103/PhysRevB.99.024403 for compositional and structural details at atomic resolution using HAADF, ABF, and EELS techniques.

[37] H. S. Horowitz, J. M. Longo, C. Booth, and C. Cases, Inorganic Syntheses 22, 73 (1983).

[38] R. Cortés-Gil, A. Arroyo, L. Ruiz-González, J. M. Alonso, A. Hernando, J. M. González-Calbet, and M. Vallet-Regí, J. Phys. Chem. Solids 67, 579 (2006).

[39] A. Varela, S. de Dios, M. Parras, M. Hernando, M. T. Fernández-Díaz, A. R. Landa-Cánovas, and J. M. GonzálezCalbet, J. Am. Chem. Soc. 131, 8660 (2009).

[40] R. Seshadri, A. Maignan, M. Herviu, N. Nguyen, and B. Raveau, Solid State Comm. 101, 453 (1997).

[41] M. H. Ehsani, M. Jalali Mehrabad, P. Kameli, M. E. Ghazi, and F. S. Razavi, J. Low Temp. Phys. 183, 359 (2016).

[42] X. J. Chen, Z. L. Zhang, J. S. Gardener, J. L. Sarrao, and C. C. Almasan, Phys. Rev. B 68, 064405 (2003).

[43] R. Suryanarayanan, G. Dhalenne, A. Revcolevschi, W. Prellier, J.-P. Renard, C. Dupas, W. Caliebe, and T. Chatterji, Solid State Comm. 113, 267 (2000).

[44] P. Yu, M. Cardona, Fundamentals of Semiconductors: Physics and Materials Properties (Springer, New York, 2010).

[45] S. Petit, M. Hennion, F. Moussa, D. Lamago, A. Ivanov, Y. M. Mukovskii, and D. Shulyatev, Phys. Rev. Lett. 102, 207201 (2009).

[46] See Supplemental Material at http://link.aps.org/supplemental/ 10.1103/PhysRevB.99.024403 for details of low-temperature electrical conductance in polycrystalline samples.

[47] C. Zener, Phys. Rev. 81, 440 (1951).

[48] C. Zener, Phys. Rev. 82, 403 (1951).

[49] P. Crespo, J. M. González, A. Hernando, and F. Yndurain, Phys. Rev. B 69, 012403 (2004).

[50] C. L. Zhang, X. J. Chen, C. C. Almasan, J. S. Gardner, and J. L. Sarrao, Phys. Rev. B 65, 134439 (2002). 